\title{
Identification and Characterization of Bioactive Components in Datura stramonium Leaves: an insight into Drugs Discovery
}

\section{*10KPASHI, VE; ${ }^{1}$ OYO-ITA, EEA; ${ }^{1} \mathrm{JONES}, \mathrm{BB} ;{ }^{2} \mathrm{OBETEN}, \mathrm{UN}{ }^{1} \mathrm{UCHO}, \mathrm{KM}$ ${ }^{3}$ OFOELO, LI}

\author{
${ }^{* 1}$ Department of Biochemistry, University of Cross River, Calabar-Nigeria \\ ${ }^{2}$ Department of Biochemistry, Alex Ekwueme Federal University Ndufu-Alike, \\ P.M.B. 1010, Abakaliki, Ebonyi State, Nigeria. \\ ${ }^{3}$ Department of Biochemistry, University of Nigeria, Nsukka, Nigeria \\ *Corresponding Author Email: vic2reshu@gmail.com
}

\begin{abstract}
Medicines from plants help in treating ailments, but to utilize them effectively in the management of diseases requires the identification of potent phytochemicals relative to conventional drugs. These phytochemicals are compared with synthetics drugs in line with their treatment regimen. An investigation was designed to identify, and characterized the different phytochemicals in Datura stramonium (Jimson weed) leaves and compared them with conventional or standard drugs. The identified phytochemicals were blasted on the drugs bank website to find their correlation and relativity. GC/MS technique was used to analyze the phytochemicals. The results showed 80 different phytochemicals belonging to several categories of phytochemicals - alkaloids, flavonoids, terpenoids, saponins, amine, and steroids. The flavonoid class had $-1.24 \%$ of $5 \mathrm{H}$-Dibenzo[c, f] [1, 2] diazepine, 3, 8 dichloro-6,11-dihydro, at 3.702 RT, Alkaloid class has - 2.98\% 2,6-Dibromobenzoquinone was detected at 4.403 RT, steroid - 2.98\% Acetanilide, 2chloro-4'-nitro- at 4.403 RT was obtained and Terpene $-2.05 \%$ of Methyl-beta.-[N-methylanilino]acrylate was detected at 4.719 RT, respectively. Most of the identified phytochemicals matched with synthetic drugs and confirmed the purpose of their applicability in traditional medicine. Considering the presence of numerous components and their correlation with conventional drugs, one can infer that this plant species has a good therapeutic application and can be utilized for health benefits.
\end{abstract}

\section{DOI:https://dx.doi.org/10.4314/jasem.v25i10.7}

Copyright: Copyright (C) 2021 Okpashi et al. This is an open access article distributed under the Creative Commons Attribution License (CCL), which permits unrestricted use, distribution, and reproduction in any medium, provided the original work is properly cited.

Dates: Received: 22 August 2021; Revised: 17 September 2021; Accepted: 06 October 2021

Keywords: Phytochemicals, Drug-bank, pharmacology, secondary-metabolites, synthetic-drug.

There are several reports about the health benefits of herbal medicines. A lot has been tested on animal models as randomized trials in managing and controlling different ailments such as diabetes mellitus, arthritis, ulcer, cancers, and cases of flu, dysentery, and diarrhea. At most, the researcher may implicate the curative effect of the plant extract to the existence of several bioactive including alkaloids, flavonoids, terpenes, steroids, saponins, amines, and alcohols. Considering that each of the listed phytochemicals has sub-compound or classes of compounds, one may wonder which particular type or classes of these phytochemicals could ameliorate the effects of a disease on the test organism. To bridge the gap of generalizing the implication of the plant's extract, this work investigated the phytochemicals in Datura stramonium (Linn) leaves (Okpashi et al., 2020). The phytochemicals were identified, quantified, and characterized. The identified phytochemicals were blasted on the conventional or synthetic drug bank website to match their correlations and relativity. Meanwhile, synthetic drugs have descriptions of formulation, synthesis, and indication for application. This plant was chosen or selected because of its applications in various fields. The plant itself is very toxic with leaves, seeds, and fruits, which limit its utilization as herbal therapy. This also caused the therapist to assume or perceived that only a small therapeutic dose can be utilized, compared to a toxic dose. Curiously, it's been used as an esoteric cannabinoid in some parts of Nigeria due to its intoxication. The learning of natural products in the expansion of curative interaction includes aspects of stereo-chemistry, biochemistry, biosynthesis, bioinformatics, and biological accomplishment to providing pathologically useful compounds. Primary metabolites are plant compounds that are expressed continuously (Jamal et al., 2016 cited in Babiker et al., 2017). Datura stramonium is known as Jimson weed (Lee, 2007). It is usually grown in recently disturbed areas and is often invasive especially in waste-dumped sites, which explain the abundance of many phytochemicals. Solanaceae that is rich in primary metabolites is a weed belonging to the Apiaceae. It has been described by the World Health Organization (WHO) as one whose many of its parts contain substances that can be used for the synthesis of useful drugs. The demand for medicinal plants is aggregating because of the rising recognition of regular products (Tatini and Raja, 2017). Plants chemicals are non- 
nutrient bioactive mixtures in plant parts. Phytochemicals are a defensive and blocking mediator against many deteriorating infections including aging, and inflammation (Debasis et al., 2015). People have been exploring plants products in pursuit of novel medicines. This has led to the use of a wide quantity of curative plants to treat various ailments. The leaves of D. stramonium are used in asthma treatment (Pretorius and Marx, 2006; Savithramma et al., 2007). The vital naturally active constituents in Datura stramonium comprised of alkaloids, atropine, and scopolamine. Atropine has been utilized in treating Parkinson's disease, peptic ulcers, diarrhea, and bronchial asthma (Ivancheva et al., 2006). Its vegetation mucilages and PolyVinyl Pyrrolidone mixture has been used as matrix-forming substances for the continual production of matrix remedies (Ahad et al., 2012). D. stramonium is a normal source of antioxidants and phytochemicals with antimicrobial activities (Akharaiyi, 2011). Its juice usually expresses considerable antimicrobial activity against several microorganisms including Staphylococcus aureus, Proteus Vulgaris, Pseudomonas aeruginosa, Escherichia coli, Aspergillus niger, and Fusarium species (Reddy, 2009). The secondary metabolites of D. stramonium are vastly active against dissimilar ailments such as antidiabetic, antiviral, etc. (Nain et al., 2013). Water extract also shows insecticidal activities (Fan and Kriton, 2005). Datura stramonium is applied in Ayurvedic drugs (Gaire and Subedi, 2013). The ethanol juice shows potent antimicrobial activities than water extracts. The leaves extracts suggest better efficacy than stem and root extract (Gachande and Khillare, 2013). In India, about $75 \%$ of the prescriptions are plants based (Solomon, 2015). The investigation on plant's natural products continues for the realizing several original energetic secondary metabolites (Ramendra and Vishnu, 2014), which have antifungal, antibacterial, and anticancer activities. The basic extracts and uncontaminated compounds isolated from plant species are applied in herbal and traditional medications. Currently, it is necessary to isolate, identify and characterize novel secondary metabolites for the treatment of diverse maladies (Jalal 2016). The unidentified organic compounds in a complex mixture can be determined through the interpretation and matching of their spectra with reference spectra (Rahim et al., 2018). The present work was carried out to identify some of the bioactive components in the leaves extract of Datura stramonium and matched with the reference spectra for nascent drug discovery, production of drugs, and proper therapeutic regiment.

\section{MATERIALS AND METHODS}

Collection of plant sample: Fresh and mature Datura stramonium leave (fruit stage), were obtained from Boki Local Government Area (LGA) of Cross River State, Nigeria. The fresh leaves were identified by Dr. Ekpene Solomon in the Department of Biological
Sciences, Cross River University of Technology, Calabar. The leaves were washed with running water and rinsed with distilled water. It was chopped into pieces and air-dried for 21 days at room temperature. The dried samples were coarse using a blender. The coarse samples were stored at room temperature for two days before extraction.

Preparation of Plant Extract: Twenty-five grams $(25 \mathrm{~g})$ of the coarse leaves were weighed and transferred into the thimbles of the soxhlet extractor, One hundred and fifty $(150 \mathrm{ml})$ normal-hexane) was measured and transferred into the round bottom flask of the soxhlet extractor. The solvent was heated to reflux through the heating mantle. After the extraction, the extracts were concentrated using a rotor for five days.

Screening of the Extract with GC/MS: A Gas Chromatography (Agilent 6890) was armed with a straight a deactivated $2 \mathrm{~mm}$ injector and $15 \mathrm{~m}$ All-tech EC-5 column ( $250 \mu$ I.D., $0.25 \mu$ film thickness). A split injection was used to inject the sample. The split ratio was set - 10:1. The oven temperature starts at 35 ${ }^{\circ} \mathrm{C}$, holds for 2 to 5 minutes, and ramped from $20{ }^{\circ} \mathrm{C}$ to $30{ }^{\circ} \mathrm{C}$. The helium gas carrier was at a $2 \mathrm{ml} /$ minute flow rate. A GC mate II bench-top double-focusing magnetic sector was operated in electron ionization (EI) mode. TSS-20001 software was used for the analyses. Low-resolution mass spectra were attained at a determining power of 1000 (20\% height definition), while scanning starts from $\mathrm{m} / \mathrm{z} 25$ to $\mathrm{m} / \mathrm{z} 700$ at 0.3 seconds per scan with a 0.2 -second inter-scan delay. High-resolution mass spectra were achieved at a resolving power of 5000 (20\% height definition) with a scanning of the magnet from $\mathrm{m} / \mathrm{z} 65$ to $\mathrm{m} / \mathrm{z} 750$ at 1 second per scan. The identification of the bioactive components of the pure compounds was matching their logged spectra with the data bank mass spectra of NIST library V 11 provided by the software of the instrument. During the analysis, the following conditions apply to the use of GC/MS techniques: GC/MS-QP2010 Agilent 6890 Plus; Ion source temperature: $200.00^{\circ} \mathrm{C}$; Interface temperature: 250.00 ${ }^{\circ}$; Solvent cut time: $2.50 \mathrm{~min}$; Detector gain mode: MS; Detector gain: $0.00 \mathrm{kV}$; Threshold: 2000; Column oven initial temperature: $70.0^{\circ} \mathrm{C}$; Injection final temperature: $250.00^{\circ} \mathrm{C}$; Injection Mode: Split; Flow control mode: linear velocity; Pressure: 116.9 $\mathrm{kPa}$, total Flow: $40.8 \mathrm{ml} \mathrm{min-1}$; Column flow: $1.80 \mathrm{ml}$ min-1; Linear velocity: $49.2 \mathrm{~cm} \mathrm{sec-1;} \mathrm{Trap} \mathrm{and}$ purge flow: $3.0 \mathrm{ml} \mathrm{min-1;} \mathrm{Split} \mathrm{Ratio:} \mathrm{20.0;} \mathrm{High-}$ pressure injection: OFF; Carrier Gas: Helium; Splitter hold: OFF.; While oven rating was as follows: Oven Temp. Program Rate Temperature $\left({ }^{\circ} \mathrm{C}\right)$ Hold Time (min) Initial: 0.00 70.0 0.00 Final: 10.0 280 5.00.

\section{RESULTS AND DISCUSSION}

Bioactive components detected in D. stramonium leaves extract: Results of bioactive analysis of Datura 
Stramonium leaves are presented in Tables designated as Table 1a, b, c, d, e, f, g, and h, separately.

Table 1a. Bioactive Profile of Datura stramonium leaves Screened with GC-MS

\begin{tabular}{|c|c|c|c|c|c|c|c|}
\hline Peak H. & RT & Area \% & Library/ID & Metabolites & Ref no & CAS & Min. Q \\
\hline 1 & 3.702 & 1.24 & $\begin{array}{l}\text { 5H-Dibenzo[c,f][1,2]diazepine, } 3,8 \\
\text { dichloro-6,11-dihydro- } \\
\text { Acetyl chloride, (2,4-dichlorophenoxy)- } \\
\text { [5-(5-Bromopyridin-3-yl)-2H-1,2,4- } \\
\text { triazol-3-yl]acetic acid }\end{array}$ & $\begin{array}{l}\text { Flavonoid } \\
- \\
\text { Flavonoid }\end{array}$ & $\begin{array}{l}124275 \\
100763 \\
142388\end{array}$ & $\begin{array}{l}000955-66-8 \\
000774-74-3 \\
1000387-64-2\end{array}$ & $\begin{array}{l}74 \\
64 \\
48\end{array}$ \\
\hline 2 & 3.834 & 1.29 & $\begin{array}{l}\text { 1,5-Hexadiene, 1,1,2,5,6,6-hexachl oro- } \\
\text { 5-Bromo-2,3-dimethoxy-6-nitrobenzene } \\
\text { aldehyde } \\
2,2^{\prime}, 4^{\prime}, 5^{\prime} \text {-Tetrachloroacetanilide }\end{array}$ & $\begin{array}{l}\text { Terpenoid } \\
\text { Flavonoid } \\
\text { Flavonoid }\end{array}$ & $\begin{array}{l}146542 \\
149053 \\
131828\end{array}$ & $\begin{array}{l}098141-62-9 \\
1000253-65-8 \\
023595-42-8\end{array}$ & $\begin{array}{l}62 \\
43 \\
35\end{array}$ \\
\hline 3 & 4.236 & 0.96 & $\begin{array}{l}\text { 2-Oxo-3-[4-bromophenyl]propanoic acid } \\
\text { s-Triazole-3-carboxaldehyde, 5-(p- } \\
\text { chlorophenyl) } \\
\text { 3-Bromo-4-chloro-5-methylbenzene } \\
\text { sulfonic acid }\end{array}$ & $\begin{array}{l}\text { Flavonoid } \\
- \\
\text { Flavonoid }\end{array}$ & $\begin{array}{l}104693 \\
71809 \\
144540\end{array}$ & $\begin{array}{l}038712-59-3 \\
026899-27-4 \\
1000305-64-9\end{array}$ & $\begin{array}{l}40 \\
35 \\
35\end{array}$ \\
\hline 4 & 4.329 & 1.50 & $\begin{array}{l}\text { 1H-Tetrazole, 1-ethyl-5-phenyl- } \\
\text { Acetamide, 2-[4-(4-bromophenyl- } \\
\text { thiazolyl]- } \\
\text { 1,3,5-triazine-2-amine, 4-chloro-N-(4- } \\
\text { ethenylphenyl)-6-methoxy- }\end{array}$ & $\begin{array}{l}\text { Flavonoid } \\
\text { Alkaloid } \\
\text { Alkaloid }\end{array}$ & $\begin{array}{l}43503 \\
154793 \\
122371\end{array}$ & $\begin{array}{l}024433-71-4 \\
017969-16-3 \\
1000401-58-8\end{array}$ & $\begin{array}{l}53 \\
51 \\
51\end{array}$ \\
\hline 5 & 4.403 & 2.98 & $\begin{array}{l}\text { 2,6-Dibromobenzoquinone } \\
\text { Ethyl 5-[2-pyridyl]-4- } \\
\text { bromopyrazolcarboxylate } \\
\text { Acetanilide, 2-chloro-4'-nitro- }\end{array}$ & $\begin{array}{l}\text { Alkaloid } \\
\text { Pyrazole } \\
\text { alkaloids } \\
\text { Steroid }\end{array}$ & $\begin{array}{l}125049 \\
154049 \\
78571\end{array}$ & $\begin{array}{l}019643-45-9 \\
1000211-49-9 \\
017329-87-2\end{array}$ & $\begin{array}{l}47 \\
38 \\
35\end{array}$ \\
\hline 6 & 4.719 & 2.05 & $\begin{array}{l}\text { Methyl .beta.-[N-methylanilino]acrylate } \\
\text { Methyl 2,4-tridecadiynoate } \\
\text { Tetryl }\end{array}$ & $\begin{array}{l}\text { Terpene } \\
\text { Flavonoid } \\
\text { Alkaloid }\end{array}$ & $\begin{array}{l}56745 \\
83274 \\
147304\end{array}$ & $\begin{array}{l}084591-20-8 \\
1000336-39-6 \\
000479-45-8\end{array}$ & $\begin{array}{l}25 \\
18 \\
15\end{array}$ \\
\hline 7 & 4.818 & 1.38 & $\begin{array}{l}\text { Benzenesulfinic acid,4-chloro-Oxazolidine, } \\
\text { 2-isopropyl-4-[2-allyli]phenoxy]methyl]- } \\
\text { Boron, difluoro(1,3-diphenyl-1,3- } \\
\text { propanediol to)- }\end{array}$ & $\begin{array}{l}\text { Alkaloid } \\
\text { Alkaloid }\end{array}$ & $\begin{array}{l}44721 \\
135472 \\
132302\end{array}$ & $\begin{array}{l}000100-03-8 \\
070687-97-7 \\
014947-61-6\end{array}$ & $\begin{array}{l}35 \\
30 \\
25\end{array}$ \\
\hline
\end{tabular}

Table 1 b. Bioactive Profile of Datura stramonium leaves Screened with GC-MS

\begin{tabular}{|c|c|c|c|c|c|c|c|}
\hline Peak H & RT & Area \% & Library/ID & Metabolites & Ref no & CAS & Min. Qual. \\
\hline 8 & 4.892 & 7.68 & $\begin{array}{l}\text { 4-benzoxazole, 2-(trifluoromethyl } \\
\text { )- } \\
\text { Pyridine, 2-(1-methyl ethyl)- } \\
\text { Pyrazine, ethenyl- }\end{array}$ & $\begin{array}{l}\text { Alcohol } \\
\text { Amide } \\
\text { Alkaloid } \\
\text { Pyrazine }\end{array}$ & $\begin{array}{l}67709 \\
9818 \\
5132\end{array}$ & $\begin{array}{l}1000396-05-4 \\
000644-98-4 \\
004177-16-6\end{array}$ & $\begin{array}{l}47 \\
41 \\
35\end{array}$ \\
\hline 9 & 5.008 & 1.08 & $\begin{array}{l}\text { Ethyl 5-[4-pyridyl]-4- } \\
\text { bromopyrazol- carboxylate } \\
\text { Pyrrole-3-carboxaldehyde, 1-(4- } \\
\text { bro } \\
\text { mo-3-methyl phenyl)-2,5- } \\
\text { dimethyl- Veratramide }\end{array}$ & $\begin{array}{l}\text { Alkaloid } \\
\text { Alkaloid } \\
\text { Alkaloid }\end{array}$ & $\begin{array}{l}154050 \\
150489 \\
49053\end{array}$ & $\begin{array}{l}1000211-51-2 \\
347331-84-4 \\
001521-39-7\end{array}$ & $\begin{array}{l}25 \\
25 \\
25\end{array}$ \\
\hline 10 & 5.178 & 0.95 & $\begin{array}{l}\text { 8-(2,3-Dimethylanilino)naphtho- } \\
\text { 1,2-quinone } \\
\text { Acetamide, 2-chloro-N-(2,3- } \\
\text { dihydro -1-methyl-pyrrolo[2,3- } \\
\text { b]quinolin-4-yl)- } \\
\text { Dimethyl trans,trans-3-(4-cyano- } \\
\text { buta-1,3-dienyl)isoxazole-4,5- } \\
\text { dicarboxylate }\end{array}$ & $\begin{array}{l}\text { Alkaloid } \\
\text { Alkaloid } \\
\text { Alkaloid }\end{array}$ & $\begin{array}{l}137361 \\
135285 \\
122365\end{array}$ & $\begin{array}{l}1000058-06-7 \\
351073-49-9 \\
1000147-02-5\end{array}$ & $\begin{array}{l}50 \\
48\end{array}$ \\
\hline 11 & 5.210 & 0.94 & $\begin{array}{l}\text { Terephthalonitrile N, N'-dioxide } \\
\text { 5-Bromo-6-methoxy-2-methyl-8- } \\
\text { nitroquinoline } \\
\text { 4,5,6-Trichloro-2- } \\
\text { benzoxazolinone }\end{array}$ & $\begin{array}{l}\text { Alkaloid } \\
\text { Alkaloid } \\
\text { Alkaloid }\end{array}$ & $\begin{array}{l}32504 \\
154789 \\
99549\end{array}$ & $\begin{array}{l}003729-34-8 \\
1000214-70-0 \\
050995-94-3\end{array}$ & $\begin{array}{l}92 \\
38 \\
35\end{array}$ \\
\hline 12 & 5.320 & 2.66 & $\begin{array}{l}\text { Benzene, 1-azido-4-nitro-Methyl } \\
\text {.beta.-[N-methylanilino]acrylate } \\
\text { Tetryl }\end{array}$ & $\begin{array}{l}\text { Alkaloid } \\
\text { Alkaloid } \\
\text { Alkaloid }\end{array}$ & $\begin{array}{l}35302 \\
56745 \\
147304\end{array}$ & $\begin{array}{l}001516-60-5 \\
084591-20-8 \\
000479-45-8\end{array}$ & $\begin{array}{l}30 \\
25 \\
12\end{array}$ \\
\hline 13 & 5.609 & 1.09 & $\begin{array}{l}\text { 1,3,4-Oxadiazol-2-amine, 5-(4- } \\
\text { bromophenyl)- } \\
\text { Terephthalonitrile N, N'-dioxide } \\
\text { 3(2H)-Isoquinolinone, 1-amino-, } \\
\text { oxime }\end{array}$ & $\begin{array}{l}\text { Amine } \\
\text { Flavonoid } \\
\text { Flavonoid }\end{array}$ & $\begin{array}{l}101482 \\
32504 \\
44125\end{array}$ & $\begin{array}{l}033621-62-4 \\
003729-34-8 \\
041536-79-2\end{array}$ & $\begin{array}{l}55 \\
53 \\
53\end{array}$ \\
\hline 14 & 5.641 & 1.41 & $\begin{array}{l}\text { s-Triazole-3-carboxaldehyde, 5- } \\
\text { chlorophenyl)- } \\
\text { 2-Methyl-2,3-epoxy-2,3-dihydro- } \\
\text { naphthoquinone } \\
\text { 4-Phenyl-2-(pyrrolidine-2-yl)-1H- } \\
\text { imidazole }\end{array}$ & $\begin{array}{l}\text { Flavonoid } \\
\text { Alkaloid } \\
\text { Alkaloid }\end{array}$ & $\begin{array}{l}71809 \\
54382 \\
77220\end{array}$ & $\begin{array}{l}026899-27-4 \\
015448-59-6 \\
944030-47-1\end{array}$ & $\begin{array}{l}50 \\
46 \\
44\end{array}$ \\
\hline
\end{tabular}

The Tables are indicated with peaks numbers (peak height), retention time (chromatogram peak number), area percentage (analyte concentration), library identified analytes (detected chemicals), bioactive 
classes (secondary metabolites), reference number, CAS numbers, and minimum quality. About 80 variable bioactive were qualitatively and quantitatively detected in $D$. stramonium leaves with different concentrations. In most instances, three bioactive of the same or different metabolites will have the same peak height and area concentration, but different retention time, reference number, and CAS number. For example, Table 1a has 5HDibenz,f][1,2]diazepine, 3, 8, dichloro-6, 11-dihydro whose metabolite is flavonoid had $1.24 \%$ area concentration at 3.702 retention time (minutes) on peak 1. A similar arrangement follows with other bioactive presented in Table $1 \mathrm{~b}$ to $1 \mathrm{~h}$.

Table 1 c. Bioactive Profile of Datura stramonium leaves Screened with GC-MS

\begin{tabular}{|c|c|c|c|c|c|c|c|}
\hline Peak H. & RT & Area \% & Library/ID & Metabolites & Ref no & CAS & Min. Qual. \\
\hline 15 & 5.670 & 0.94 & $\begin{array}{l}\text { Ethane, 1-[(2-chloroethyl)thio]-2- } \\
\text { (ethylthio)- } \\
\text { s-Triazole-3- } \\
\text { carboxaldehyde,chlorophenyl)- } \\
\text { Methyl 5,6-dichloropyridine-3-carb }\end{array}$ & $\begin{array}{l}\text { Alkane } \\
\text { Flavonoid } \\
\text { Alkaloid }\end{array}$ & $\begin{array}{l}51593 \\
71809 \\
69825\end{array}$ & $\begin{array}{l}092569-22-7 \\
5026899-27-4 \\
056055-54-0\end{array}$ & $\begin{array}{l}90 \\
47 \\
45\end{array}$ \\
\hline 16 & 5.696 & 0.92 & $\begin{array}{l}\text { s-Triazole-3-carboxaldehyde, 5- } \\
\text { chlorophenyl)- } \\
\text { Furazan, nitrophenyl-, 5-oxide } \\
\text { 5-Bromo-6-methoxy-2-methyl-8- } \\
\text { nitroquinoline }\end{array}$ & $\begin{array}{l}\text { Flavonoid } \\
\text { Steroid } \\
\text { isoquinoline al } \\
\text { kaloid }\end{array}$ & $\begin{array}{l}71809 \\
71753 \\
154789\end{array}$ & $\begin{array}{l}026899-27-4 \\
049558-03-4 \\
1000214-70-0\end{array}$ & $\begin{array}{l}92 \\
56 \\
53\end{array}$ \\
\hline 17 & 5.837 & 1.67 & $\begin{array}{l}\text { Benzenesulfinic acid, 4-chloro-1H- } \\
\text { Tetrazole, 1-ethyl-5-phenyl- } \\
\text { 5-Methyl-4-[4-(1,2,4-triazole-1-ylm } \\
\text { ethyl)phenyl]-1,2,4-triazole-3-thiol }\end{array}$ & $\begin{array}{l}\text { Flavonoid } \\
\text { Alkaloid } \\
\text { Alkaloid }\end{array}$ & $\begin{array}{l}44721 \\
43503 \\
131973\end{array}$ & $\begin{array}{l}000100-03-8 \\
024433-71-4 \\
1000410-40-8\end{array}$ & $\begin{array}{l}53 \\
53 \\
49\end{array}$ \\
\hline 18 & 6.027 & 1.73 & $\begin{array}{l}\text { 9,10-Di[chloromethyl]-S- } \\
\text { octahydroanthracene } \\
\text { 5-Bromo-6-methoxy-2-methyl-8- } \\
\text { nitroquinoline } \\
\text { 1,2-Butadiene, 1,1,4-trichloro- }\end{array}$ & $\begin{array}{l}\text { Terpenoid } \\
\text { Quinoline alka } \\
\text { loid } \\
\text { Terpene }\end{array}$ & $\begin{array}{l}141786 \\
154789 \\
29487\end{array}$ & $\begin{array}{l}018256-06-9 \\
1000214-70-0 \\
058679-08-6\end{array}$ & $\begin{array}{l}53 \\
38 \\
35\end{array}$ \\
\hline 19 & 6.052 & 1.09 & $\begin{array}{l}\text { N-(2-Phenylethyl)undeca-(2Z,4E)-diene- } \\
\text { 8,10-diynamide } \\
\text { 5-Chloro-N-methylisatoic anhydrazide } \\
\text { 1,2-Butadiene, 1,1,4-trichloro- }\end{array}$ & $\begin{array}{l}\text { Amide } \\
\text { Amide } \\
\text { Terpene }\end{array}$ & $\begin{array}{l}137413 \\
75605 \\
29487\end{array}$ & $\begin{array}{l}099615-80-2 \\
040707-01-5 \\
058679-08-6\end{array}$ & $\begin{array}{l}38 \\
35 \\
35\end{array}$ \\
\hline 20 & 6.558 & 1.12 & $\begin{array}{l}\text { s-Triazole-3- } \\
\text { carboxaldehyde,chlorophenyl)- } \\
\text { 2-Methyl-2,3-epoxy-2,3-dihydro-1,4- } \\
\text { naphthoquinone } \\
\text { 1,2-Digermacyclopentane, 1,1,2,2- } \\
\text { tetramethyl- }\end{array}$ & $\begin{array}{l}\text { Flavonoid } \\
\text { Quinoline alka } \\
\text { loid } \\
\text { Cyclic Alkane }\end{array}$ & $\begin{array}{l}71809 \\
54382 \\
111878\end{array}$ & $\begin{array}{l}5-026899-27-4 \\
015448-59-6 \\
035839-71-5\end{array}$ & $\begin{array}{l}68 \\
55 \\
53\end{array}$ \\
\hline 21 & 6.587 & 1.22 & $\begin{array}{l}\text { 5-Bromo-6-methoxy-2-methyl-8- } \\
\text { nitroquinoline } \\
\text { Mercury, chloromethyl- } \\
\text { N-(2-Phenylethyl)undeca-(2Z,4E)-di } \\
\text { ene-8,10-diynamide }\end{array}$ & $\begin{array}{l}\text { Quinoline alka } \\
\text { loid } \\
\text { Alkane } \\
\text { Amide }\end{array}$ & $\begin{array}{l}154789 \\
113828 \\
137413\end{array}$ & $\begin{array}{l}1000214-70-0 \\
000115-09-3 \\
099615-80-2\end{array}$ & $\begin{array}{l}62 \\
59 \\
38\end{array}$ \\
\hline
\end{tabular}

Table 1 d. Bioactive Profile of Datura stramonium leaves Screened with GC-MS

\begin{tabular}{|c|c|c|c|c|c|c|c|}
\hline Peak H. & RT & Area \% & Library/ID & Metabolites & Ref no & CAS & $\begin{array}{l}\text { Min. } \\
\text { Quality }\end{array}$ \\
\hline \multirow[t]{3}{*}{22} & 6.648 & 1.20 & $\begin{array}{l}\text { 9,10-Di[chloromethyl]-S-octahydroanthracene } \\
\text { 4,6-Dibromo-2-benzoxazolinone } 3,5,6,7,7,8 \text { - }\end{array}$ & Alkaloid & 141786 & $018256-06-9$ & 43 \\
\hline & & & Hexachloro-5,6,7,8-tetrahydro-S-triazolo[4,3- & Alkaloid & 150985 & $1000260-92-6$ & 38 \\
\hline & & & a]pyridine & Alkaloid & 185439 & $022841-85-6$ & 38 \\
\hline \multirow[t]{3}{*}{23} & 6.947 & 1.14 & Terephthalonitrile N, N'-dioxide & Steroidal Alkaloid & 32504 & $003729-34-8$ & 50 \\
\hline & & & Androstan-4,16-dien-3-one, 17-formyl- & Steroid & 157994 & $114724-34-4$ & 38 \\
\hline & & & Acetanilide, 2-chloro-4'-nitro- & Steroidal Alkaloid & 78571 & $017329-87-2$ & 35 \\
\hline \multirow[t]{3}{*}{24} & 6.989 & 0.95 & 1,4-Dioxaspiro[4.5]deca-6,9-diene-2,8-dione & Terpene & 37021 & $004385-47-1$ & 50 \\
\hline & & & $\begin{array}{l}\text { 6-Bromo-4,7-dimethoxy-2H-1,3-benzo dioxole-5- } \\
\text { carbaldehyde }\end{array}$ & Aldehyde & 147355 & $109548-10-9$ & 38 \\
\hline & & & 2-Thiophenecarbonitrile, 4-Bromo- & Steroidal Alkaloid & 53820 & $1000362-65-0$ & 35 \\
\hline \multirow[t]{4}{*}{25} & 7.053 & 1.30 & Methyl 2-bromo-3-cyano-6-methylpyr idine-4- & Alkaloid & 115716 & $1000410-58-8$ & 55 \\
\hline & & & carboxylate & Alkaloid & 141694 & $1000111-66-8$ & 47 \\
\hline & & & $\begin{array}{l}\text { 7,8-Methylenedioxy-5-oxo-1-fluorenecarboxylic } \\
\text { acid, methyl ester }\end{array}$ & Alkaloid & 145855 & $1000260-14-0$ & 45 \\
\hline & & & $\begin{array}{l}\text { 2,3-Diazabicyclo[3.3.0]octa-3,7-diene-4- } \\
\text { carboxylic acid, 2-(4-methoxyphenyl)-, ethyl ester }\end{array}$ & & & & \\
\hline \multirow[t]{3}{*}{26} & 7.069 & 1.72 & Acetanilide, 2-chloro-4'-nitro- & Alkaloid & 78571 & $017329-87-2$ & 35 \\
\hline & & & 7-[2-Chloroethyl]guanine & Steroid Nucleotide & 77363 & $022247-87-6$ & 35 \\
\hline & & & 5-Bromo-6-methoxy-2-methyl-8-nitroquinoline & Isoquinoline & 154789 & $1000214-70-0$ & 35 \\
\hline \multirow[t]{3}{*}{27} & 7.120 & 1.70 & (3-Nitro-benzyl)-O-tolyl-amine & Amine Alkaloid & 103988 & $1000296-75-0$ & 56 \\
\hline & & & 4-Amino-6-morpholino-5-nitropyrimidine & Saponin Saponin & 88749 & $024957-88-8$ & 45 \\
\hline & & & Phenol, 2-cyclohexyl-4,6-dinitro- & & 125969 & $000131-89-5$ & 44 \\
\hline \multirow[t]{3}{*}{28} & 7.644 & 1.06 & (3-Nitro-benzyl)-O-tolyl-amine & Amine & 103988 & $1000296-75-0$ & 90 \\
\hline & & & [(2-Oxochromen-4-yl)sulfanyl]acetic acid & Flavonoid & 97970 & $1000410-90-7$ & 40 \\
\hline & & & 5-Chloro-3-[(2-chloro-acetylamino $)$ & Amine alkaloid & 136837 & $1000294-79-5$ & 40 \\
\hline
\end{tabular}

Table 1 e. Bioactive Profile of Datura stramonium leaves Screened with GC-MS 


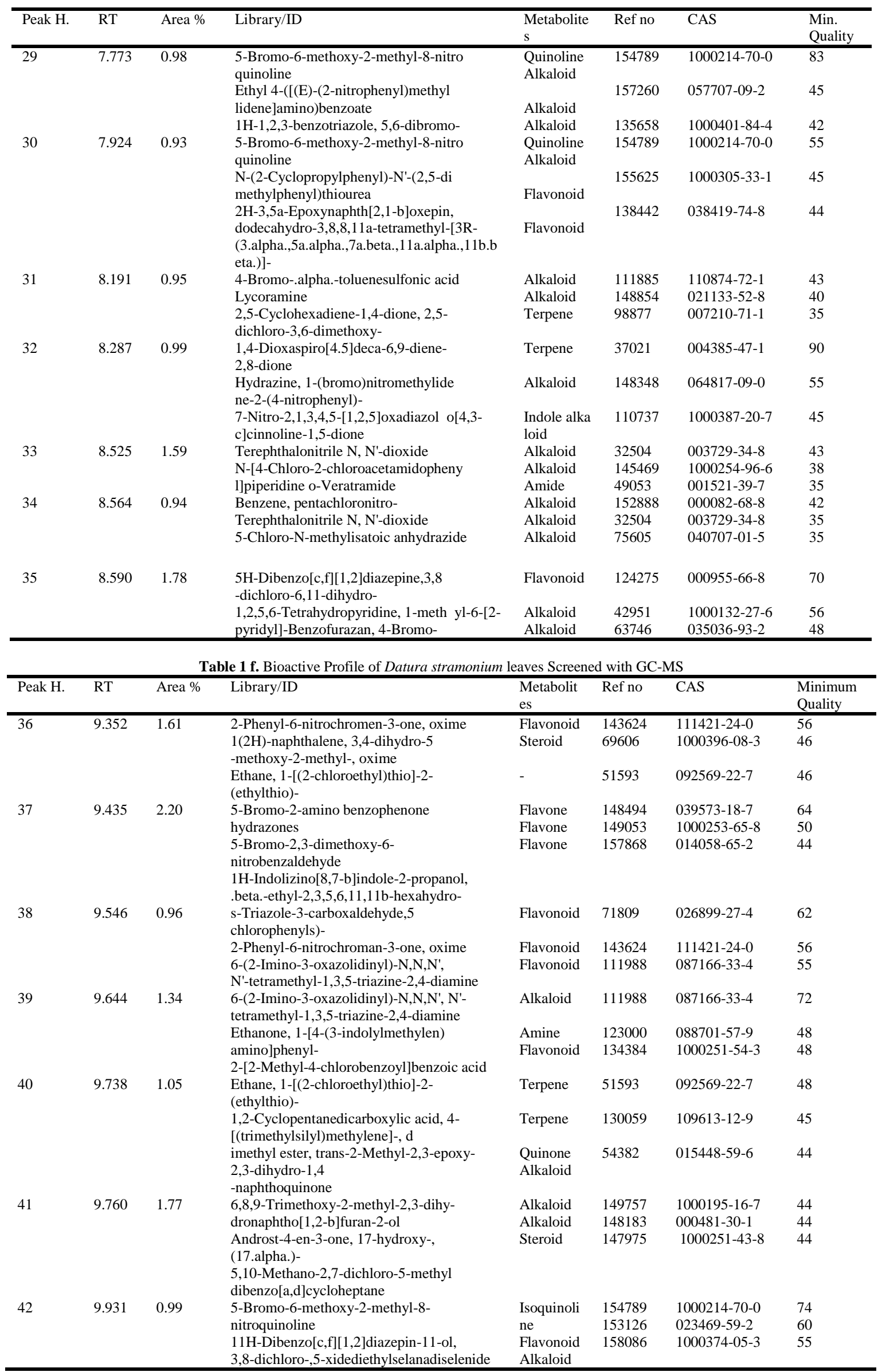


Table 1 g. Bioactive Profile of Datura stramonium leaves Screened with GC-MS

\begin{tabular}{|c|c|c|c|c|c|c|c|}
\hline Peak H. & RT & Area \% & Library/ID & Metabolites & Ref no & CAS & $\begin{array}{l}\text { Minimum } \\
\text { Quality }\end{array}$ \\
\hline 43 & 9.988 & 1.21 & $\begin{array}{l}\text { 4-(1-Benzofuran-2-yl)-7- } \\
\text { methoxychromen-2-one } \\
\text { 6-Bromo-4,7-dimethoxy-2H-1,3- } \\
\text { benzodioxole-5-carbaldehyde } \\
\text { Pyrrolo[2,3-b]indole, 1-benzoyl-1, } \\
\text { 2,3,3a,8,8a-hexahydro-3a,8-dimethy } \\
\text { 1-, (3aS-cis)- }\end{array}$ & $\begin{array}{l}\text { Flavon } \\
\text { Flavonoid } \\
\text { Alkaloid }\end{array}$ & $\begin{array}{l}151822 \\
147355 \\
151993\end{array}$ & $\begin{array}{l}108154-51-4 \\
109548-10-9 \\
054833-65-7\end{array}$ & $\begin{array}{l}44 \\
25 \\
25\end{array}$ \\
\hline 44 & 10.001 & 1.25 & $\begin{array}{l}\text { 2,3,5-Trichlorophenol, O- } \\
\text { trifluoroacetyl- } \\
\text { 2,4,6-Trichlorophenol, trifluoroacetate } \\
\text { 2-Chloro-5-methyl-4,6-bis(2-thienyl) } \\
\text { pyrimidine }\end{array}$ & $\begin{array}{l}\text { Alkaloid } \\
\text { Flavonol } \\
\text { Amide }\end{array}$ & $\begin{array}{l}152147 \\
152146 \\
151235\end{array}$ & $\begin{array}{l}1000374-26-9 \\
1000365-26-5 \\
131022-82-7\end{array}$ & $\begin{array}{l}30 \\
30 \\
25\end{array}$ \\
\hline 45 & 10.310 & 1.38 & $\begin{array}{l}\text { Ethyl 4-([(E)-(2- } \\
\text { nitrophenyl)methylidene] } \\
\text { amino)benzoate } \\
\text { 1H-Indolizino[8,7-b]indole-1-propanol, } \\
\text {.beta.-ethyl-2,3,5,6,11,11b-hexahydro- } \\
\text { Chromone, 5-hydroxy-6,7,8- } \\
\text { trimethoxy-2,3-dimethyl- }\end{array}$ & $\begin{array}{l}\text { Flavonoid } \\
\text { Flavonoid } \\
\text { Flavonoid }\end{array}$ & $\begin{array}{l}157260 \\
157867 \\
139558\end{array}$ & $\begin{array}{l}057707-09-2 \\
055670-04-7 \\
1000124-95-9\end{array}$ & $\begin{array}{l}25 \\
25 \\
15\end{array}$ \\
\hline 46 & 11.039 & 1.43 & $\begin{array}{l}\text { 1H-Benzotriazole, 4,5,6,7-tetrachloro- } \\
\text { 3-(3,4-Methylenedioxy)phenyl-4- } \\
\text { nitrocyclohexanone } \\
\text { 4-Methyl-6-phenyl-3-thioxo-3,4- } \\
\text { dihydro-1,2,4-triazine-5(2H)-one }\end{array}$ & $\begin{array}{l}\text { Flavonoid } \\
\text { Flavone } \\
\text { Alkaloid }\end{array}$ & $\begin{array}{l}116404 \\
123534 \\
82127\end{array}$ & $\begin{array}{l}002338-10-5 \\
1000111-64-4 \\
022936-87-4\end{array}$ & $\begin{array}{l}47 \\
46 \\
45\end{array}$ \\
\hline 47 & 11.037 & 2.06 & $\begin{array}{l}\text { Carbazol-1-ol, 1,2,3,4-tetrahydro-6- } \\
\text { Bromo-9-ethyl- } \\
\text { Ethyl 4-Bromo-alpha-cyano-beta- } \\
\text { methyl-cis-cinnamate } \\
\text { Benzene, pentachloronitro- }\end{array}$ & $\begin{array}{l}\text { Flavonoid } \\
\text { Terpenoid } \\
\text { Terpenoid }\end{array}$ & $\begin{array}{l}152409 \\
152287 \\
152889\end{array}$ & $\begin{array}{l}1000263-26-5 \\
020992-89-6 \\
000082-68-8\end{array}$ & $\begin{array}{l}90 \\
58 \\
56\end{array}$ \\
\hline 48 & 11.069 & 1.06 & $\begin{array}{l}\text { Pyrazole, 1-methyl-3-(4-nitrophenyl)- } \\
\text { 1,3,4-Oxadiazol-2-amine, 5-(4- } \\
\text { bromophenyl)- } \\
\text { 1H-Tetrazole, 1-ethyl-5-phenyl- }\end{array}$ & $\begin{array}{l}\text { Alkaloid } \\
\text { Alkaloid } \\
\text { Alkaloid }\end{array}$ & $\begin{array}{l}67254 \\
101482 \\
43503\end{array}$ & $\begin{array}{r}073387-59-4 \\
033621-62-4 \\
024433-71-4\end{array}$ & $\begin{array}{l}58 \\
55 \\
53\end{array}$ \\
\hline 49 & 11.339 & 1.45 & $\begin{array}{l}\text { 4-(4-Chlorophenyl)-3- } \\
\text { morpholinopyrrol-2-carbaldehyde } \\
\text { Dibenzo[b,f][1,4]diazocine, 5,6,11,12- } \\
\text { tetrahydro-2-(trifluoromethyl) } \\
\text { Ethanone, 1-(3,5-bromophenol)- }\end{array}$ & $\begin{array}{l}\text { Flavonoid } \\
\text { Flavonoid } \\
\text { Flavonoid }\end{array}$ & $\begin{array}{l}149595 \\
137963 \\
136769\end{array}$ & $\begin{array}{l}142405-54-7 \\
027188-36-9 \\
014401-73-1\end{array}$ & $\begin{array}{l}46 \\
46 \\
45\end{array}$ \\
\hline
\end{tabular}

Table 1 h. Bioactive Profile of Datura stramonium leaves Screened with GC-MS

\begin{tabular}{|c|c|c|c|c|c|c|c|}
\hline Peak H. & RT & Area \% & Library/ID & Metabolites & Ref no & CAS & $\begin{array}{l}\text { Minimum } \\
\text { Quality }\end{array}$ \\
\hline 50 & 11.664 & 1.33 & $\begin{array}{l}\text { Mercury, chloromethyl- } \\
\text { 5-Bromo-6-methoxy-2-methyl-8-nitroquinoline } \\
\text { 4,5,6-Trichloro-2-benzoxazolinone }\end{array}$ & $\begin{array}{l}\text { Alkaloid } \\
\text { Isoquinolin } \\
\text { e } \\
\text { Isoquinolin } \\
\text { e }\end{array}$ & $\begin{array}{l}113828 \\
154789 \\
99549\end{array}$ & $\begin{array}{l}000115-09-3 \\
1000214-70-0 \\
050995-94-3\end{array}$ & $\begin{array}{l}80 \\
62 \\
62\end{array}$ \\
\hline 51 & 11.850 & 1.03 & $\begin{array}{l}\text { 3-Bromo-2,5-dichlorothiophene 5-Bromo-6- } \\
\text { methoxy-2-methyl-8-nitroquinoline } \\
\text { 3,5-Dichloro-2-hydrazinopyridine }\end{array}$ & $\begin{array}{l}\text { Flavonoid } \\
\text { Quinoline } \\
\text { Alkaloid }\end{array}$ & $\begin{array}{l}93483 \\
154789 \\
45439\end{array}$ & $\begin{array}{l}060404-18-4 \\
1000214-70-0 \\
104408-23-3\end{array}$ & $\begin{array}{l}40 \\
38 \\
30\end{array}$ \\
\hline 52 & 13.229 & 1.01 & $\begin{array}{l}\text { Pyrazole, 1-methyl-3-(4-nitrophenyl)- } \\
\text { 1H-Tetrazole, 1-ethyl-5-phenyl- 1,3,4- } \\
\text { Oxadiazol-2-amine, 5-(4-bromophenyl)- }\end{array}$ & $\begin{array}{l}\text { Alkaloid } \\
\text { Alkaloid }\end{array}$ & $\begin{array}{l}67254 \\
43503 \\
101482\end{array}$ & $\begin{array}{l}073387-59-4 \\
024433-71-4 \\
033621-62-4\end{array}$ & $\begin{array}{l}59 \\
53 \\
46\end{array}$ \\
\hline 53 & 13.911 & 1.25 & $\begin{array}{l}\text { 1,3,4-Oxadiazol-2-amine,5-(4-bromophenyl)- } \\
\text { 1H-Tetrazole, 1-ethyl-5-phenyl- } \\
\text { s-Triazole-3-carboxaldehyde, } 5 \text { chlorophenyls)- }\end{array}$ & $\begin{array}{l}\text { Flavonoid } \\
\text { Alkaloid } \\
\text { Alkaloid }\end{array}$ & $\begin{array}{l}101482 \\
43503 \\
71809\end{array}$ & $\begin{array}{l}033621-62-4 \\
024433-71-4 \\
026899-27-4\end{array}$ & $\begin{array}{l}55 \\
53 \\
50\end{array}$ \\
\hline 54 & 14.126 & 21.66 & $\begin{array}{l}\text { Phytol, 4-Chloro-6,7-dimethyl-3-N-(oxolan- } \\
\text { 2-ylmethyl)-2H-pyrrolo[3,4-c]pyridine-1,3- } \\
\text { diimine } \\
\text { 2,2,6-Trimethyl-1-(3-methyl beta-1, 3-dienyl)-7- } \\
\text { oxabicyclo[4.1.0]heptan-3-ol }\end{array}$ & $\begin{array}{l}\text { Alkaloid } \\
\text { Alkaloid } \\
\text { Flavonol }\end{array}$ & $\begin{array}{l}155850 \\
151308 \\
85557\end{array}$ & $\begin{array}{l}1000388-01-6 \\
1000191-85-4\end{array}$ & $\begin{array}{l}41 \\
41\end{array}$ \\
\hline
\end{tabular}

An investigation to identify, quantifies, and characterized the different bioactive compounds in Datura stramonium (Jimson weed) leaves was carried out. The results showed 80 different bioactive constituents belonging to different metabolites including alkaloids, flavonoids, terpenoids, saponins, amine, and steroids. Several phytochemicals were detected and quantified - flavonoid had - $5 \mathrm{H}$ Dibenzo[c,f][1,2]diazepine $-1.24 \%$ concentration at
3.702 retention time see Table $1 \mathrm{a}$. This compound is also called 3-amino-5,12,12a-trihydro-4-oxo1Hpyrazolo[4,3-e] thiochromeno [4,3-c] [1,2] diazepines (Ramendra and Vishnu, 2014). 5HDibenzodiazepines are used in treating an array of health problems. They act by activating a sedative substance in the brain and central nervous system (CNS). Negative outcomes may include dizziness, poor coordination, and depression (Salzman, 1990). 
5H-Dibenzodiazepines are usually used for the temporary management of severe insomnia. 5HDibenzodiazepines remain a potent anticonvulsant and vastly effective at averting protracted epileptic seizures. The dangerous part of this leave extract is when used in combination with alcohol or opioids (American Psychiatric Association, 1998). 5HDibenzodiazepines bind stereo-specifically to an exclusive portion of GABA receptors with large protein complexes, located at some neurons in the CNS. GABA is the main inhibitory neurotransmitter in the brain (Stahl, 2002). 5H-Dibenzodiazepines potentiate GABA-mediated transmission and are indirect GABA agonists (Buffett-Jerrott and Stewart, 2002; Fick et al., 2003). A chemical class of terpenoid - 1,5-Hexadiene, 1,1,2,5,6,6-hexachloro was detected and shown in Table 1a. It has a $1.29 \%$ concentration and a 3.834 retention time (RT). Also, an alkaloid 2,6-Dibromobenzoquinone commonly called Quinone, 2,6-dibromo- had 2.98\% at 4.403 RT. 2,6Dibromoquinone-4-chloroimide is a reagent for the determination of phenols (Wagner et al., 2007). Katherine (2016) study the effect of halobenzoquinone on human neural stem cells (hNSCs), a flow cytometric analysis revealed that hNSCs exposed to $0.5 \mu \mathrm{M}$ of 2,6-dichlorobenzoquinone (2, 6- DCBQ), for 96 hours which occasioned greater quantities of cells in S-phase. This proposes the arrest of the cell cycle in the S-phase where deoxyribonucleic (DNA) replication ensues. In Table 1b, 4-benzoxazole, 2(trifluoromethyl ) which belongs to the Chlorzoxazone family of drugs was detected in the $D$. stramonium leave extract. Its concentration was $7.68 \%$ at 4.892 RT. This class of chemical is an alcohol derivative that acts as a muscle relaxant bearing tranquilizing properties. It is claimed to prevent muscle twinge by causing an effect mostly on the spinal cord and subcortical areas (Martindale, https://www.drugbank.ca/drugs/DB00356). A series of ten different oxadiazole analogs were appraised for their in vitro activities against cancer in a single-dose assay. The oxadiazole equivalents exhibited reasonable activity against cancer on several cell lines. The oxadiazole_analogs increase their anticancer activities (Mohamed et al., 2013). Another alkaloid 4-Phenyl-2-(pyrrolidine-2-yl)-1H-imidazole whose IUPAC name is 5-phenyl-1H-imidazole was detected and estimated as $1.41 \%$ at 5.641 RT. 5-phenyl-1Himidazole $4.41 \%$ at 5.641 RT; 1,3,4-Oxadiazol-2amine $1.09 \%$ at 5.609 RT and s-Triazole-3carboxaldehyde, 5-chlorophenyl were detected. There are supplemented as azole antifungal agents. They work by obstructing the making of ergosterol, a vital constituent of cell membranes in fungal. Its action is by disrupting the cytochrome p450 51 (Lanosterol 14alpha demethylase) in fungal. This is crucial in the structure of the cell membranes of fungus. Its inhibition resulted in cell lysis (Tassaneeyakul et al., 1998). The inhibition in the production of ergosterol, causes holes to appear in the cell membrane. This is because cell membranes are necessary for the survival of fungi. Their general functions include Steroid hydroxylase action, which breaks down more than a few carcinogens, tablets, and diluents to reactive metabolites (Tassaneeyakul et al., 1998; Monostory et al., 2004). Furazan, nitrophenyl-, 5-oxide $0.92 \%$ at 5.696 RT. This compound is an organic compound nitrobenzenes. They contain a nitrobenzene moiety, this bioactive plays a vital role in metalloaminopeptidase activity by removing the $\mathrm{N}$ terminal of methionine from an emerging protein. The $\mathrm{N}$-terminal of methionine is repeatedly sliced when the second residue in the primary sequence is lesser and uncharged (Met-Ala-, Cys, Gly, Pro) Berman et al. (2000). 5 -Bromo-6-methoxy-2-methyl-8nitroquinoline (Quinoline alkaloid) $1.22 \%$ at 6.587 RT. This is an organic compound known as nitroquinolines. It contains a nitro group bonded to a quinoline (Pelletier et al., 1994) see Table 1c. This phytochemical had exhibits antitumor activity via inhibiting the type-2 methionine of aminopeptidase (MetAP2) protein involved in angiogenesis. Its antibacterial action originates from the metal ion complexion that is useful for bacterial growth (Pelletier et al., 1995; Shim et al., 2010). In Table 1d, the most important bioactive were detected and estimated. For example, androstane-4, 16-dien-3one,17-formyl $1.14 \%$ at $6.947 \mathrm{RT}$ is categorize as androstanes. This compound belongs to androgens and derivatives, they are 3-hydroxylated C19 steroid hormones. Known to service the development of masculine characteristics, this accounted for its utilization as an esoteric cannabinoid by some youths (Chen et al., 2000). These same properties corroborate the use of this plant extract for the treatment of hair loss in humans, and function in Steroid hormone receptors - ligand-activated transcription factors that control the expression of a eukaryotic gene and affect cellular proliferation and differentiation in target tissues (Takahashi et al., 2004). 2, 5-Cyclohexadiene1,4-dione with $0.95 \%$ at $8.191 \mathrm{RT}$ is also called RH-1. These are organic compounds known as pbenzoquinones. Benzoquinones have two $\mathrm{C}=\mathrm{O}$ groups attached to carbon 1- and 4-positions, respectively. RH-1 has been used in trials, to study the handling of Progressive Hard Cancers and Non-Hodgkin's Lymphoma (Tudor et al., 2005). At the superoxide dismutase activity, the enzyme helps as a quinone reductase by linking with conjugation reactions of hydroquinone that are involved in detoxification corridors and biosynthetic routes including the vitamin (Overington et al., 2006; Imming et al., 2006). 1]piperidine o-Veratramide $1.59 \%$ at $8.525 \mathrm{RT}$. This compound belongs to aminopiperidines. They contain piperidine that carries an amino group. At the triglyceride lipase activity pathway, l]piperidine is applied in the decontamination of xenobiotics and activation prodrugs containing ester and amide. In Table 1e, tricyclic dibenzodiazepine, categorized as an uncommon antipsychotic agent

$(5 \mathrm{H}-$ 
Dibenzo[c,f][1,2]diazepine,3,-dichloro-6,11-dihydro) was detected and quantified $-1.78 \%$ at 8.590 RT. This compound binds to some receptors in the central nervous system and displays a distinctive pharmacological effect. $5 \mathrm{H}$ Dibenzo[c,f][1,2]diazepine is a serotonin antagonist, with high binding to 5 -HT $2 \mathrm{~A} / 2 \mathrm{C}$ receptor subtype (Berman et al., 2000; Weizman et al., 2003; ). It also displays high affinity to numerous dopaminergic receptors but expresses weak antagonism at the dopamine D2 receptor, a receptor that controls neuroleptic activity (Guarrera, 1999). The major adverse effect associated with the administration of this agent is agranulocytosis (an acute febrile condition noticeable by severe reduction in blood granulocytes and often linked with the use of certain drugs). Dibenzo[c,f][1,2]diazepine is a psychotropic agent belonging to benzisoxazole derivatives indicated for the treatment of schizophrenia (a mental disorder that is characterized by disturbances in thought in the case of hallucination). $5 \mathrm{H}$ - Dibenzo[c,f][1,2]diazepine is a discriminating monoaminergic antagonist with a strong affinity for the serotonin Type-2 $\left(5 \mathrm{HT}_{2}\right)$, dopamine Type-2 (D2), 1 and 2 adrenergic, and H1 histaminergic receptors (Young et al., 2004). 5HDibenzo[c,f][1,2]diazepine serves as an antagonist to other receptors sites, but with lesser potency. Antagonism at receptors other than dopamine and $5 \mathrm{HT}_{2}$ with similar receptor affinities explain the side effect of 5H-Dibenzo[c,f][1,2] diazepines (Stonehouse and Jones, 2005). 5H-Dibenzo[c,f][1,2]diazepine's antagonism of muscarinic M1-5 receptors explains its anticholinergic outcome after administration or ingestion. $\quad 5 \mathrm{H}$-Dibenzo[c,f][1,2]diazepine's antagonism of histamine $\mathrm{H} 1$ receptors elucidate the somnolence experience with this drug. 5HDibenzo[c,f][1,2]diazepine's antagonism of adrenergic-1 receptors could clarify the orthostatic hypotension observed with this bioactive (Takano et al., 2006). 5H-Dibenzo[c,f][1,2]diazepine's antipsychotic action is prospectively regulated via a combination of antagonistic effects at D2 receptors in the mesolimbic pathway and 5-HT2A receptors in the frontal cortex (Chen et al., 2002). The D-2 antagonism could relieve a helpful symptom while 5-HT2A antagonism alleviates harmful symptoms.

A $1.61 \%$ at $9.352 \mathrm{RT}$ of $1(2 \mathrm{H})$-naphthalenone, 3,4dihydro-5 was detected. It is called 2-[4-(4Chlorophenyl) Cyclohexylidene]-3,4-Dihydroxy1(2h)-Naphthalene. Its mechanism of action deals with ubiquinone binding to catalyzes the transformation of dihydroorotate to orotate, while quinone will remain an electron acceptor (Berman et al., 2000). Phenyl-2Hchromene derivatives are derivatives to synthesize triazole and biotin-containing chromene derivatives, to facilitate the purification of protein targets (Bhaskar et al., 2010). These organic compounds are phenol ethers. They are aromatic compounds having an ether group substituted with a benzene ring. It derivatives is 6-(2-phenoxy ethoxy)-1, 3, 5-triazine-2, 4-diamine. Its function deals with the acetylation of the coenzyme-A carboxylase complex. Whereat first, biotin carboxylase will catalyze the carboxylation of the carrier protein and then the transcarboxylase transfers the $\mathrm{Ca}^{+}$(Berman et al., 2000), find Table 1f. A flavonoid named 4-(1-Benzofuran-2-yl)-7methoxychromen-2-one had $1.21 \%$ at 9.988 RT was detected in D. stramonium leaves. This compound is a flavone whose backbone is 2-phenylchromen-4-one (2-phenyl-1-benzopyran-4-one) (Shimada et al., 2009). It has antibiotic activity (for Gram-positive bacteria) and antitumor activity (for some mouse tumors). It binds non-covalently to a chromophore which is the cytotoxic and mutagenic component of the antibiotic. The chromophore in turn binds to DNA as a weak intercalator and reasons a single - and doublestrand breakdown (Shimada et al., 2010). 2-Chloro-5methyl-4, 6-bis (2-thienyl) pyrimidine $1.25 \%$ at $10.001 \mathrm{RT}$ was obtained from $D$. stramonium leaves and presented in Table 1g. This organic compound is known as aminobenzenesulfonamides (Derewlany et $a l ., 1994)$. They contain benzenesulfonamide moiety with an amine group bonded to the benzene ring. This amide is directed for the treatment of bacterial infections which cause bronchitis, prostatitis, and urinary tract infections. The role of 2-Chloro-5methyl-4,6-bis(2-thienyl) pyrimidine is to inhibit the enzymatic conversion of pteridine and paminobenzoic acid (PABA) to dihydropteroate acid by opposing PABA from binding to dihydrofolate synthetase, an intermediate of tetrahydrofolic acid (THF) synthesis. THF is usually needed to synthesize purines and dTMP. Any disruption of its synthesis will inhibit the growth of bacterial. Pyrimethamine and trimethoprim inhibit dihydrofolate reductase, additional pace in THF synthesis, and act in synergy with 2-Chloro-5-methyl-4,6-bis(2-thienyl) pyrimidine. 2-Chloro-5-methyl-4,6-bis(2-thienyl) pyrimidine as a side effect which may be nausea, vomiting, diarrhea, and hypersensitivity reactions (Friaza et al., 2010). Hematologic effects such as anemia, agranulocytosis, thrombocytopenia, and hemolytic anemia in patients with glucose-6phosphate dehydrogenase insufficiency may arise (Bratlid and Bergan, 1976). 2-Chloro-5-methyl-4,6bis(2-thienyl) pyrimidine might dislodge bilirubin from albumin binding sites triggering jaundice or kernicterus in newborns (Angelakou et al., 1993). In Table 1h, 5-Bromo-6-methoxy-2-methyl-8nitroquinoline $1.33 \%$ at $11.664 \mathrm{RT}$ was obtained. This compound is nitroquinolines and their derivatives. They contain a nitro group bonded to a quinoline. It is indicated for dealing with Schistosomiasis affected by Schistosoma mansoni (Filho et al., 2006). 5Bromo-6-methoxy-2-methyl-8-nitroquinoline is an anthelmintic with schistosomicidal activity against Schistosoma mansoni, but not against other Schistosoma spp. 5-Bromo-6-methoxy-2-methyl-8nitroquinoline causes worms to move from the mesenteric veins to the liver where the male worms are 
retained; the female worms return to the mesentery, but can no longer release eggs (Overington et al., 2006). 5-Bromo-6-methoxy-2-methyl-8nitroquinoline may link with an irreversible inhibitor of nucleic acid metabolism. A premise has been put forth that the drug is activated by a single step, in which a schistosome sulfotransferase enzyme converts 5-Bromo-6-methoxy-2-methyl-8-nitroquinoline into an ester (probably acetate, phosphate, or sulfate group). Successively, the ester suddenly dissociates, the resultant electrophilic reactant is capable of alkylating the schistosome DNA (Imming et al., 2006; Pica-Mattoccia et al., 2006). The phytochemistry and therapeutic elucidation of Datura stramonium leave extract have been well recognized in this investigation. Because of its multiple uses, more bioactive screening and structural elucidation studies are yet to be explored. The information presented in this work would help promote research aiming at the development of methods for isolation and application of new agents for medical applications and agroindustries based on natural products derived from plants.

Conclusion: The information about jimson weed (leaves) covers many aspects including botanical, chemical, pharmaceutical, and medical. The objectives of this study were to (a) develop an improved GC-MS procedure for the analysis of bioactive components of jimson weed leaves to show the known and unknown alkaloids, Flavonoids, Terpenoids, saponins, amide, amines, and alcohols using the GC-MS technique. These bioactive were identified, classified, characterized, and estimated. They were blasted against the synthetic drug bank to ascertain their therapeutic relevance, correlation, and relativity. Much of their pharmacological relevance was describe together with their mechanism of action. This was performed with the hope that drugs producers, researchers, and herbal technicians will find a better understanding in redirecting their treatment synthesis of novel therapies.

Competing interests: Authors have declared that no competing interests exist. The products used for this research are commonly and predominantly use products in our area of research and country. There is no conflict of interest between the authors and producers of the products because we do not intend to use these products as an avenue for any litigation but the advancement of knowledge. Also, the research was not funded by the producing company rather it was funded by the personal efforts of the authors.

\section{REFERENCES}

Ahad, HA; Babu, UA; Nagesh, K; Kiran, DS; Madhavi, KB (2012). Fabrication of glimepiride Datura stramonium leaves mucilage and polyvinyl pyrrolidone sustained release matrix tablets: in vitro evaluation. Kathmandu university jof sci. engn. \& Tech. 8(1):63-72.

Akharaiyi, FC (2011). Antibacterial, Phytochemical and Antioxidant activities of Datura metal. Int. J. Pharm Tech Research; 3(1):478-483.

American Psychiatric Association (1998). Practice guideline for the treatment of patients with panic disorder. Am J Psychiatry. 155(5):1-34.

Angelakou, A; Valsami, G; Koupparis, M; Macheras, $P$ (1993). Use of 1-anilino-8 naphthalene sulphonate as an ion probe for the potentiometric study of the binding of sulphonamides to bovine serum albumin and plasma. J Pharm Pharma. 45(5):434-8.

Babiker, F; Jamal, P; Mirghani, MES; Ansari, AH (2017). Characterization, purification, and identification of some Alkaloids in Datura stramonium. International Food Research Journal, 24(Suppl): S540-S543

Berman, HM; Westbrook, J; Feng, Z; Gilliland, G; Bhat, TN; Weissig, H; Shindyalov, IN; Bourne, PE (2000). The Protein Data Bank. Nucleic Acids Res. 28(1):235-42.

Bhaskar, CD; Seetaram, M; Philip, DC; Sabita, N; Sakkarapalayam, M; Todd, E (2010). Synthesis of Function-Oriented 2-Phenyl-2H-chromene Derivatives Using L-Pipecolinic Acid and Substituted Guanidine Organocatalysts. Tetrahedron Letters 51(19):2567-2570.

Bratlid, D; Bergan, T (1976). Displacement of albumin-bound antimicrobial agents by bilirubin, Pharmacology. 14(5):464-72.

Buffett-Jerrott, SE; Stewart, SH; (2002). Cognitive and sedative effects of benzodiazepine use. Curr Pharm Des. 8:45-58.

Chen, X; Ji, ZL; Chen, YZ (2002). Therapeutic Target Database, Nucleic Acids Res. 30(1):412-5.

Debasis, S; Sunil, KR; Hemlata, G; Hasi. RD (2015). A novel coumarin derivative, 8-methoxy chrome2-one alleviates collagen-induced arthritis by down-regulating nitric oxide, $\mathrm{NF} \kappa \mathrm{B}$, and proinflammatory cytokines. International Immunopharmacology.

Derewlany, LO; Knie, B; Koren, G (1994). Arylamine $\mathrm{N}$-acetyltransferase activity of the human placenta. J Pharmacol Exp Ther. 269(2):756-60.

Fan, D; John, J; Kriton, KH (2005). Effects of lyphosate, chlorsulfuron, and methyl jasmonate 
on growth and alkaloid biosynthesis of Jimson weed (Datura stramonium L.) Pestic.Biochem.Physiol.84 (2):155

Fick, DM; Cooper, JW; Wade, WE (2003). Updating the Beers criteria for potentially inappropriate medication use in older adults: results of a US consensus panel of experts. Arch Intern Med.163:2716-2724.

Filho, RP; de-Souza, MCM; Pinto, PL; Paula, GA; Brandt, CA; da-Silveira, MA (2007). Design, synthesis, and in vivo evaluation of oxamniquine methacrylate and acrylamide prodrugs. Bioorg Med Chem. 15(3):1229-36.

Friaza, V; Morilla, R; Respaldiza, N; de-laHorra, C; Calderon, EJ (2010). Pneumocystis jiroveci dihydropteroate synthase gene mutations among colonized individuals and Pneumocystis pneumonia patients from Spain. Postgrad Med. 122(6):24-8.

Gachande, BD; Khillare, EM (2013). In-vitro evaluation of Datura species for potential antimicrobial activity. Bioscience Discovery; 4(1):78-81.

Gaire, BP; Subedi, L (2013). A review on the pharmacological and toxicological aspects of Datura stramonium L. Journal of Integrative Medicine; 11(2): 73-9.

Guarrera, PM (1999). Traditional antihelmintic, antiparasitic and repellent uses of plants in Central Italy. Journal of Ethnopharmacology; 68(13):183-192.

Imming, P; Sinning, C; Meyer, A (2006). Drugs, their targets, and the nature and number of drug targets. Nat Rev Drug Discov. 5(10):821-34.

Ivancheva, S; Nikolova, M; Tsvetkova, R (2006). Pharmacological activities and biologically active compounds of Bulgarian medicinal plants, Phytochemistry, Adva. Rese. 87-103

Jalal, HM (2016). Biological activities importance of Tetrazole derivatives. European Academic Research. 3(12); p. 12796 - 2804

Jamal, P; Akbar, I; Hashim, YZH; Jaswir, I (2016). Process development for maximum lycopene production from selected fruit waste and its antioxidant and antiradical activity. Journal of Food Processing and Technology 7(4): 1-7.

Katherine, ZF (2016). Effects of Halobenzoquinone Water Disinfection By-Products on Human Neural Stem Cells. A thesis submitted for the degree of Master of Science. Department of Laboratory Medicine and Pathology, University of Alberta. Pp. 4.

Lee, M (2007). Solanaceae IV: Atropa belladonna, deadly nightshade. The Journal of the Royal College of Physicians of Edinburgh 37: 77-84.

Mohamed, JA; Vikram, PSR; Monika, S; Ramdayal, S; Surender, SJ; Sabina, YS; Pradeep, K (2013). Synthesis, Anticancer and Molecular Docking Studies of 2-(4-chlorophenyl)-5-aryl-1,3,4Oxadiazole Analogues. Medicinal Chemistry. 3:294-297.

Monostory, K; Hazai, E; Vereczkey, L (2004). Inhibition of cytochrome P450 enzymes participating in p-nitrophenol hydroxylation by drugs known as CYP2E1 inhibitors. Chem Biol Interact. 147(3):331-40.

Nain, J; Bhatt, S; Dhyani, S; Joshi, N (2013). Phytochemical screening of secondary metabolites of Datura stramonium, Int. J. Curr. Pharm. Res., 5(2), 151-153.

Okpashi, VE; Abeng, FE; Ushie, OA; Inyang, IH; Kate, MU (2020). Comparative Study on the differences in Elemental-Uptake by Seeds and Leaves of Datura Stramonium (Linn). Bioscience Research, 17(2): 2009-2014.

Overington, JP; Al-Lazikani, B; Hopkins, AL (2006). How many drug targets are there? Nat Rev Drug Discov. 5(12):993-6.

Pelletier, C; Prognon, P; Bourlioux, P (1995). Roles of divalent cations and $\mathrm{pH}$ in the mechanism of action of nitroxoline against Escherichia coli strains. Antimicrob Agents Chemother. 39(3):70713.

Pelletier, C; Prognon, P; Latrache, H; Villart, L; Bourlioux, P (1994). Microbiological consequences of chelation of bivalent metal cations by nitroxoline. Pathol Biol (Paris). 42(5):406-11

Pretorius, E; Marx, J (2006). Datura stramonium in asthma treatment and possible effects on prenatal development. Environ Toxicol Pharmacol; 21(3):331-7.

Rahim, E; Ismail, A; Omar, MN; Rahmat, UN; Ahmad, W (2018). GC-MS Analysis of Phytochemical Compounds in Syzygium polyanthum Leaves Extracted using UltrasoundAssisted Method. Pharmacog J. 10(1):110-9. 
Ramendra, P; Vishnu, JR (2014). Natural and Synthetic Chromenes, Fused Chromenes, and Versatility of Dihydrobenzo[h]chromenes in Organic Synthesis

Reddy, BU (2009). Antimicrobial activity of Datura stramonium L. and Tylophora indica (Burm. F.) Merr, Pharmac. Online, (1), 1293-1300.

Salzman, C (1990). Benzodiazepine dependency: summary of the APA task force on benzodiazepines. Psychopharmacol Bull. 26:6162.

Savithramma, N; Sulochana, C; Rao, KN (2007). Ethnobotanical survey of plants used to treat asthma in Andhra Pradesh, India $J$ Ethnopharmacol; 113(1):54-61.

Shim, JS; Matsui, Y; Bhat, S; Nacev, BA; Xu, J; Bhang, HE; Dhara, S; Han, KC; Chong, CR; Pomper, MG; So, A; Liu, JO (2010). Effect of nitroxoline on angiogenesis and growth of human bladder cancer. J Natl Cancer Inst. 102(24):185573.

Shimada, T; Tanaka, K; Takenaka, S; Foroozesh, MK; Murayama, N; Yamazaki, H; Guengerich, FP; Komori, M (2009). Reverse type I binding spectra of human cytochrome P450 1B1 induced by a flavonoid, stilbene, pyrene, naphthalene, phenanthrene, and biphenyl derivatives that inhibit catalytic activity: a structure-function relationship study. Chem Res Toxicol. 22(7):1325-33.

Shimada, T; Tanaka, K; Takenaka, S; Murayama, N; Martin, MV; Foroozesh, MK; Yamazaki H; Guengerich, FP; Komori, M (2010). Structurefunction relationships of inhibition of human cytochromes P450 1A1, 1A2, 1B1, 2C9, and 3A4 by 33 flavonoid derivatives. Chem Res Toxicol. 23(12):1921-35.

Solomon, G (2015). Preliminary Phytochemical Screening and in vitro Antimicrobial Activity of Datura stramonium Leaves Extracts Collected from Eastern Ethiopia. Int. Res. J. Biological Sci. 4(1), 55-59.
Stahl, SM (2002). Don't ask, don't tell, but benzodiazepines are still the leading treatments for anxiety disorder. J Clin. Psychiatry. 63:756757.

Stonehouse, AH; Jones, FS (2005). Bromocriptine and clozapine regulate dopamine 2 receptor gene expression in the mouse striatum. J Mol Neurosci. 25(1):29-36.

Takahashi, M; Tatsugi, Y; Kohno, T (2004). Endocrinological and pathological effects of anabolic-androgenic steroid in male rats. Endocr J. 51(4):425-34

Takano, A; Suhara ,T; Kusumi, I; Takahashi, Y; Asai, Y; Yasuno, F; Ichimiya, T; Inoue, M; Sudo, Y; Koyama, T (2006). Time course of dopamine D2 receptor occupancy by clozapine with medium and high plasma concentrations. Prog Neuropsychopharmacol Biol Psychiatry. 30(1):75-81.

Tassaneeyakul, W; Birkett, DJ; Miners, JO (1998). Inhibition of human hepatic cytochrome P4502E1 by azole antifungals, CNS-active drugs, and nonsteroidal anti-inflammatory agents. Xenobiotica. 28(3):293-301.

Tatini, D; Raja, C (2017). Newer Insights into the Pharmacological Activities of Datura Stramonium Linn: A Review. Indo Am. J. Pharma. Res. 7(9). P. $441-444$

Tudor, G; Alley, M; Nelson, CM; Huang, R; Covell, DG; Gutierrez, P; Sausville, EA (2005). Cytotoxicity of RH (2005). NAD (P) H: quinone acceptor oxidoreductase (NQO1)-independent oxidative stress and apoptosis induction. Anticancer Drugs. 16(4):381-91

Wagner, AK; Ross-Degnan, D; Gurwitz, JH (2007). Effect of New York regulatory action on benzodiazepine prescribing and hip fracture rates. Ann Intern Med.146:96-103.

Weizman, T; Pick, CG; Backer, MM; Rigai, T; Bloch, M; Schreiber. S (2003). The antinociceptive effect of amisulpride in mice is mediated through opioid mechanisms. Eur J Pharmacol. 478(2-3):155-9. 